\title{
SISTEMA DE DIREÇÃO ELÉTRICA ASSISTIDA COM INTELIGÊNCIA ARTIFICIAL
}

\author{
José L. N. Silvaㄹ, Mateus P. Aires, Francisco W. S. Mesquita, Jhayro A. S. Júnior e Gabriel S. \\ Fonseca. \\ ${ }^{1}$ Instituto Federal de Educação e Tecnologia do Ceará (IFCE) \\ E-mail: leonardo.silva@,ifce.edu.br
}

\section{RESUMO}

Os carros autônomos elétricos ou híbridos são o futuro da indústria automobilística, estes carros são divididos em diversos sistemas de controle eletrônico. Entre esses sistemas de controle o sistema de direção representa um desafio para o desenvolvimento dos carros autônomos. Neste trabalho é desenvolvido um protótipo de um sistema de direção assistida (Driver Assistance Systems - DAS) de acionamento elétrico (Electrically Powered Steering EPS) e proposto um algoritmo de controle da posição angular do servomecanismo baseado em lógica fuzzy. Partindo de uma bancada didática existente no laboratório que disponibiliza um sistema de direção com acionamento hidráulico (Hydraulic Power Steering - HPS) foram levantadas as curvas (mapas) de operação da direção elétrica e ajustados os parâmetros do controlador para que o sistema quando instalado em um veículo possa desempenhar a função de seguidor de trajetória em cenários de ambientes urbanos. O sistema de direção proposto neste trabalho pretende ser uma contribuição para o desenvolvimento de carros autônomos, contribuir para redução do número de acidentes e também ser um acessório opcional em veículos com motoristas humanos para situações extremas de direção (onde existe o risco a vida), como já existe atualmente nos sistemas de frenagem antitravamento (Antilock Braking System - ABS). O controlador proposto é embarcado em um módulo eletrônico e executa as funções descritas acima e está integrado com os demais sistemas embarcados nos veículos através de uma rede de troca de dados (Control Area Network - CAN), desta forma tem-se um sistema Steer-by-wire com controlador fuzzy. Os resultados das simulações computacionais mostraram que a solução proposta pode ser empregada em veículos comerciais.

\section{INTRODUÇÃO}

Os carros autônomos são o futuro da indústria automobilística, esta frase de efeito é citada em livros de robótica [1] [2] [3] e na mídia especialidade em automóveis. Grandes passos já foram dados com esse objetivo, porém esta caminhada ainda está longe do fim [4] [5] [6] [7]. Os acidentes de trânsito são a principal causa de morte entre os jovens entre 10 e 24 anos., cerca de 90\% dos acidentes de trânsito são causados por falha humana [8] [9]. Existe a necessidade de tecnologias para reduzir o número de acidentes de trânsito para preservar vidas humanas. Os sistemas de freio ABS e os sistemas de controle de estabilidade ESP já são tecnologias que atuam no veículo de forma autônoma nas situações de risco a vida[10].

Nos últimos anos, tanto o mundo acadêmico como a indústria têm-se dedicado ao desenvolvimento de Sistemas de Assistência ao Motorista (Driver Assistance Systems - DAS) 
e Veículos Autônomos (Autonomous Vehicles - AV) com o objetivo de diminuir o número de acidentes rodoviários [11]. Neste trabalho um sistema de direção é proposto como uma contribuição para o desenvolvimento de carros autônomos e também ser um acessório visando contribuir para redução do número de acidentes atuando na direção assistida e segurança veicular. Outra possível aplicação desse sistema de direção é em veículos semiautônomos com a função de piloto automático, estacionamento automático e transporte de pessoas com mobilidade reduzida. Esta integração com os demais sistemas embarcados nos veículos será possível através de uma rede CAN [12].

O controlador fuzzy para o sistema de direção quando embarcado em um veículo recebe sinais de referência (set points) de vários outros sistemas embarcados no veículo, entre eles o sistema de freio eletrônico (Brake-by-wire), aceleração eletrônica (Throttle-by-wire), sistema de estabilidade da carroçaria, sensor de ângulo de direção, sensor de torque ou barra de torção da coluna de direção. Portanto, é um controlador multivariável. Por esta razão de aplicação dos controladores clássicos monovariáveis como PID (Proporcional-Integral-Derivativo) representa uma "restrição" para sistemas Steer-by-wire [13].

Outra característica importante da operação deste controlador é o levantamento das curvas (mapas) de operação do sistema de direção que definem a maneira como o controlador responde aos sinais de entrada em função da velocidade do veículo [13][7]. Quando embarcado em um veículo autônomo este controlador fuzzy receberá referência também do gerador de trajetória para realizar a navegação autônoma observando a sinalização da via e os critérios de segurança da mobilidade urbana [2].

As contribuições tecnológicas deste trabalho são as possíveis aplicações para o controlador desenvolvido nesta pesquisa. Inicialmente as aplicações propostas são:

Piloto automático: consiste no controle longitudinal e lateral da dinâmica do veículo através do sistema de direção assistida. O problema do piloto automático de um veículo, cuja posição é determinada $x$ (distância no eixo horizontal/vertical) e $\phi$ (ângulo do veículo em relação ao eixo horizontal/vertical), as variáveis de entrada. A variável de controle (saída) é $\theta$ (posição angular do sistema de direção), supondo velocidade constante. Neste problema é necessário um gerador de trajetória para fornecer o sinal de referência (setpoint). Este gerador é baseado em um sistema de visão artificial, GPS e outros mecanismos de localização, mapeamento e navegação.

Estacionamento automático (self-parking): o problema do estacionamento de um veículo, cuja posição é determinada pelas variáveis $x$ (distância no eixo horizontal) e $\phi$ (ângulo do veículo em relação ao eixo horizontal), as variáveis de entrada. A variável de controle (saída) é $\theta$ (posição angular do sistema de direção), supondo velocidade constante. Observe-se que esta aplicação é diferente e mais complexa que a anterior (piloto automático), porque não se considera a existência de uma trajetória de referência e sim a solução do problema em tempo real pelo controlador fuzzy.

No desenvolvimento dos carros autônomos. $\mathrm{O}$ autor entende que em função do grande número de montadoras existirão no futuro várias tecnologias de carros autônomos e estas variações serão uma oportunidade para esta pesquisa. Estas variações são reflexos dos vários segmentos da indústria automobilística. Por exemplo, o sistema de transmissão automática desenvolvido pela Honda na década de 1970 foi o primeiro comercializado mundialmente, mas atualmente cada montadora tem sua própria tecnologia de transmissão automática. Portanto, não existirá apenas uma solução de sistema de direção assistida para os carros autônomos. 
Este trabalho está organizado da seguinte maneira, na seção I (Introdução) foi apresentada uma rápida descrição da tecnologia de carros autônomos e descrição da contribuição desta pesquisa nos sistemas de direção. Na seção II são apresentados os vários sistemas de controle que possibilitam um carro navegar de forma autônoma e a comunicação CAN entre esses sistemas. Dentre esses sistemas, o sistema de direção é o mais detalhado por ser o sistema a ser controlado por lógica fuzzy.

Na seção III é apresentado o controlador fuzzy proposto para o sistema direção assistida. A modelagem matemática do sistema de direção elétrica EPS, o projeto do controlador fuzzy, aspectos técnicos e detalhes da implementação para embarcar o mesmo em um microcontrolador com barramento CAN. Na seção IV são apresentados resultados da simulação computacional, a análise destes resultados, adotando como critérios erros no regime transitório e erro no regime estacionário da resposta dinâmica do sistema de controle fuzzy proposto. Na seção V são apresentadas as conclusões e perspectivas da aplicação desta tecnologia em veículos comerciais.

\section{SISTEMAS DE CONTROLE EMBARCADOS}

Neste trabalho o automóvel convencional é dividido em sete sistemas de controle como mostrado na Figura 1 e descritos abaixo:

- Sistema de potência ou motorização: inclui um motor de combustão que conta com um avançado sistema de controle de injeção de combustível e controle ignição da mistura; nos veículos híbridos esse sistema também inclui motores elétricos com controle de torque e rotação. Outra característica importante deste sistema é a aceleração com pedal eletrônico, conhecida como throttle-wire.

- Sistema de transmissão: sistema responsável pela distribuição do torque gerado no motor até as rodas. Pode ser manual ou automático. Existem diversos modelos automáticos e pesquisas na área de inteligência artificial e otimização com o objetivo de tornar esses sistemas mais eficientes e econômicos.

Estes dois primeiros sistemas se encontram em estágio de comercialização com várias soluções tecnológicas de alto desempenho. Portanto, teoricamente estão prontos para serem embarcados nos carros autônomos.

- Sistema elétrico: consiste dos circuitos de potência e controle embarcados nos veículos. Foi o sistema que mais evolui nos últimos anos, passando de um circuito analógico para um circuito digital com barramento de comunicação e realizando funções importantes para o conforto, desempenho e segurança do veículo [12].

- Sistema de suspensão: Sistema responsável pelo conforto dos passageiros é divido em sistema de suspensão passiva e ativa [7]. Este último um sistema adaptativo em função da escolha do usuário a das condições da pista de rodagem [15].

- Sistema de controle de carroceria: responsável pelo controle das portas, cintos de segurança, air bag e até ar condicionado. É um sistema mais evidente nos carros de luxo e muito importante para segurança e conforto dos passageiros. Também conta com um barramento de comunicação [7]. 


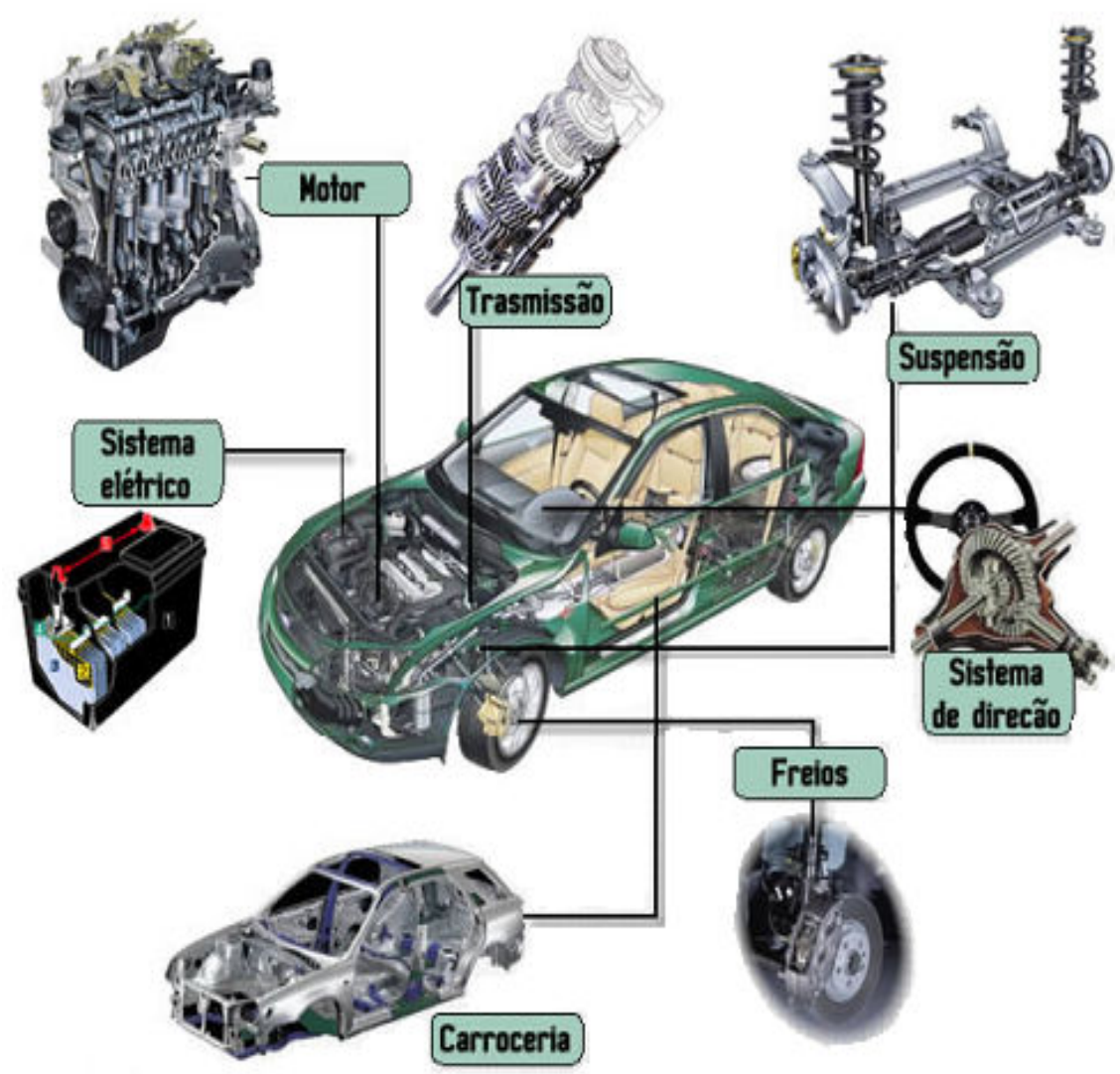

Figura 1 - Principais Sistemas de um automóvel de passeio. Fonte: Santos, 2010.

Os próximos dois sistemas representam os maiores desafios para o desenvolvimento dos carros autônomos por estarem diretamente relacionados com a segurança dos passageiros e com a navegação autônoma em ambientes urbanos.

- Sistema de frenagem ou freios: inicialmente responsável pela parada do veículo em segurança. Porém com o surgimento do sistema de frenagem $A B S$ novas funções como o controle de tração (Traction Control System - TCS) e controle de estabilidade (Electronic Stability Program - ESP) foram desenvolvidas [10][16]. Existem soluções com sistema de frenagem através de inversores de frequência em veículos híbridos e elétricos, denominada tecnologia brake-by-wire.

- Sistema de direção: responsável pelo posicionamento angular das rodas (esterçamento) em relação à carroceria do veículo. Este sistema é o objeto de estudo desta pesquisa pelo fato de representar um grande desafio para indústria automobilista de veículos autônomos [7] [15]. Na seção III é apresentada a modelagem matemática da cinemática e da dinâmica de um veiculo com o objetivo de controlar o sistema de direção. As tecnologias nesse sistema podem ser classificadas em dois grupos steer-by-wire e drive-by-wire. O primeiro trata basicamente de sistema de direção elétrica com funções auxiliares, como por exemplo, seguimento de reta e estacionamento automático (self-parking). No grupo drive-by-wire o sistema de direção é autônomo e integrador ao SLAM para executar a trajetória desejada pelo usuário [2].

Além destes sistemas, outros aplicados apenas aos carros autônomos precisam ser desenvolvidos. Como apresentado em [2] os carros autônomos realizam a localização e mapeamento simultâneos em tempo real. Esta função referenciada na literatura acadêmica 
como SLAM (Simultaneous Localization and Mapping) é uma área de pesquisa em robótica móvel muito ativo nas últimas décadas. O SLAM incorpora módulos de localização e de mapeamento que são fortemente dependentes um do outro e interligados através de uma rede de comunicação [15]. O sinal gerado pelo SLAM é enviado ao controlador de execução da trajetória desejada que determina qual o ângulo deve ser adotado nas rodas. A partir deste instante o controlador de direção atua em malha fechada para garantir a realização da ação.

Os sistemas de direção automotiva tradicionalmente são divididos em dois grupos: direção não assistida e direção assistida. No primeiro grupo estão os sistemas mecânicos tipo setor e sem fim e o sistema pinhão cremalheira adotado pela maioria das montadoras. Nos sistemas de direção assistida estão o sistema de direção com acionamento hidráulico (Hydraulic Power Steering - HPS), sistema de direção com acionamento eletro-hidráulica (Electro-Hydraulic Power Steering - EHPS) e o sistema de direção com acionamento elétrico (Electrically Powered Steering - EPS), sendo este último o estado da arte e o objeto de estudo deste trabalho.

Na direção EPS a assistência ao motorista é realizada por um motor elétrico que atua diretamente sobre o mecanismo de direção e pode ser instalado na coluna de direção, caixa de direção ou na própria cremalheira. Este sistema não tem assistência hidráulica. O sistema EPS já está disponível em alguns modelos comerciais, e é um avanço em direção ao carro completamente autônomo. A assistência ao motorista no sistema EPS começa ao girar o volante, nesse instante o sensor de torque informa a existência de um esforço na barra de torção dado pelo deslocamento angular relativo entre os extremos da barra. Simultaneamente, um sensor de ângulo da direção informa o ângulo girado pelo motorista e a velocidade com que é movimentado o volante [7].

Nos veículos comercializados com rede CAN o controle da direção elétrica é realizado em malha fechada geralmente com um controlador PID. As entradas são os sensores de ângulo de direção e sensor de torque. A saída é a corrente elétrica aplicada ao motor elétrico instalado no sistema de direção para desenvolver um torque, portanto um sistema steer-by-wire. Porém, é necessário que este controlador (módulo eletrônico) da EPS também recebe as seguintes informações: 1) velocidade do veículo, recebida do módulo de controle ABS através da rede CAN e 2) rotação do motor, recebida do módulo de controle do motor através da rede CAN. O módulo de controle da direção calcula a assistência necessária e aciona o atuador. A velocidade do veículo é utilizada para ajustar o grau de assistência a ser aplicado. O conceito básico é maior torque em baixa velocidade e menor torque em alta velocidade. Essa relação entre o torque e a velocidade do veículo é conhecida como curva de operação, mostrada na Figura 2 para um veículo comercial de baixa potência.

Com o auxílio das curvas de operação desenvolvidas na engenharia do veiculo e gravadas na memória do módulo de controle, a assistência necessária é ajustada em função da velocidade. A Figura 2 apresenta como exemplo, curvas típicas que representam os mapas de ajuste da assistência em função da velocidade de veículos comerciais das linhas leve e pesada. Reparar na diminuição do torque de assistência com o aumento da velocidade. A soma do torque aplicado ao volante pelo motorista mais o torque exercido pelo motor elétrico na barra de direção, é denominado torque efetivo que atua sobre o sistema de direção nos carros convencionais. O torque efetivo da direção assistida nos carros autônomos é somente o torque desenvolvido no atuador determinado pelo controlador. 
O uso de técnicas de controle clássico como o PID para seguinte destas curvas obrigado o uso de vários controladores PID's pelo menos um para cada região de operação descrita pela curva. Este problema não existe para o controlador fuzzy que já é projetado o seguinte destas curvas com o mesmo conjunto de parâmetros independente da região de operação.

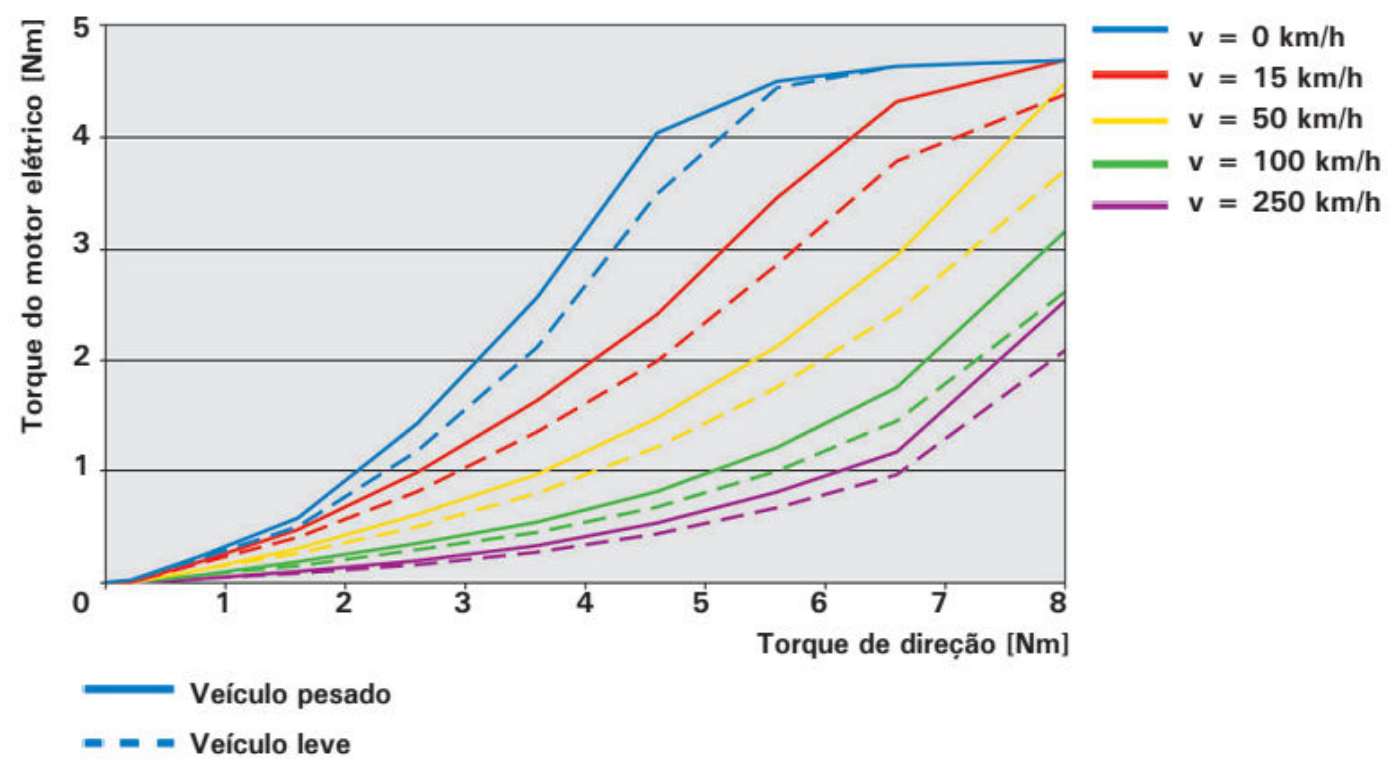

Figura 2 - Mapas de operação do EPS.

\section{SISTEMA DE CONTROLE FUZZY PROPOSTO}

A lógica fuzzy, também conhecida como lógica "nebulosa", permite o tratamento de expressões que envolvem grandezas descritas de forma não exata. Como exemplo, considerese a descrição das ações de um motorista sobre o pedal de freio de um automóvel que se aproxima de uma curva:

se $\{($ velocidade $=$ muito grande $) \wedge($ raio_de_curvatura $=$ pequeno $)\}$

então \{força_sobre pedal $=$ grande $\}$

se $\{($ velocidade $=$ mediana $) \wedge($ raio_de_curvatura $=$ grande $)\}$

então \{força_sobre_pedal = pequena $\}$

Nessas expressões, as variáveis velocidade, raio de curvatura, força sobre pedal poderiam ser numéricas, do tipo velocidade $=98,743 \mathrm{Km} / \mathrm{s}$, raio_de_curvatura $=7,184 \mathrm{~m} \mathrm{e}$ força_sobre pedal $=5,39 \mathrm{~N}$. Entretanto, instrutores de auto-escola costumam utilizar expressões que envolvem incertezas do tipo muito grande, bem devagar, pouco à esquerda e outros termos de natureza linguística. O tratamento de expressões que envolvem variáveis liguisticas pode ser realizado atraves da utilização da lógica fuzzy que, por sua vez, se baseia em conceitos da teoria de conjuntos nebulosos [3] [13].

Em [18] é apresentado um controlador fuzzy tipo Takagi-Sugeno de ordem zero embarcado em um Controlador Lógico Programável para o controle de posição de robô manipulador industrial. Também é apresentada metodologia de projeto e uma prova da estabilidade deste controlador pelo critério de Popov. Este trabalho é adotado como referência para a 
metodologia de projeto. A principal diferença está no hardware utilizado, pois para o sistema de direção EPS foi adotado um microcontrolador com barramento CAN.

Entre as várias técnicas de inteligência artificial a opção pela Lógica Fuzzy se justifica principalmente por três razões. A primeira é o fato de que o controlador do sistema de direção de um carro autônomo trabalha com informações imprecisas (nebulosas) para gerar sinais de controle. Por exemplo, um gerador de trajetória baseado em SLAM envia uma referência de ângulo para o controlador do sistema de direção, enquanto o sistema de visão envia outra referência em função da sinalização da via. A máquina de inferência fuzzy permitirá definir qual o torque deve ser aplicado à barra de direção para executar essa mudança de direção de forma satisfatória.

A segunda razão está relacionada ao número e o tipo de variáveis que são envolvidas nesta tarefa. A lógica fuzzy permite que cada variável seja modelada por um conjunto de funções e ainda na fase de projeto todos os possíveis comportamentos sejam simulados, sem a necessidade de treinamentos exaustivos e possíveis surpresas desagradáveis de comportamentos (respostas). Portanto, a lógica fuzzy permite projetar e embarcar um controlador para executar a tarefa de controle do sistema de direção assistida. E a terceira razão está relacionado a capacidade de aprendizagem do sistema. A identificação dos perfis dos usuários dos veículos através da seleção de funções fuzzy e associando a lógica com outras técnicas como Redes Neurais Artificiais para tornar o sistema cada vez mais inteligente.

Projetar um controlador fuzzy: esta etapa consiste de identificar as variáveis do sistema de sistema, modelar essas variáveis através de conjuntos fuzzy. Em seguida estabelecer as relações entre as variáveis com regras e adotar uma máquina de inferência fuzzy para gerar o sinal de controle.

O projeto do controlador fuzzy como apresentado em [18] e [19] inclui a fuzzificação de suas variáveis, funções de pertinência, regras de inferência e algoritmos de defuzzificação. Portanto, projetar um controlador fuzzy não é um jogo de tentativa e erro, é sim uma metodologia científica que visa obter o melhor desempenho de uma ação de controle. Neste trabalho as variáveis de entrada são a velocidade do veículo e o erro de posição angular da direção das rodas, a variável de saída é torque de direção aplicado na caixa de direção que leva o erro da posição angular a zero. as funções de pertinência criadas foram do tipo triangular e trapezoidal. Um conjunto com quinze regras de inferência foi adotado. A Figura 3 mostra o controlador no ambiente de desenvolvimento. 


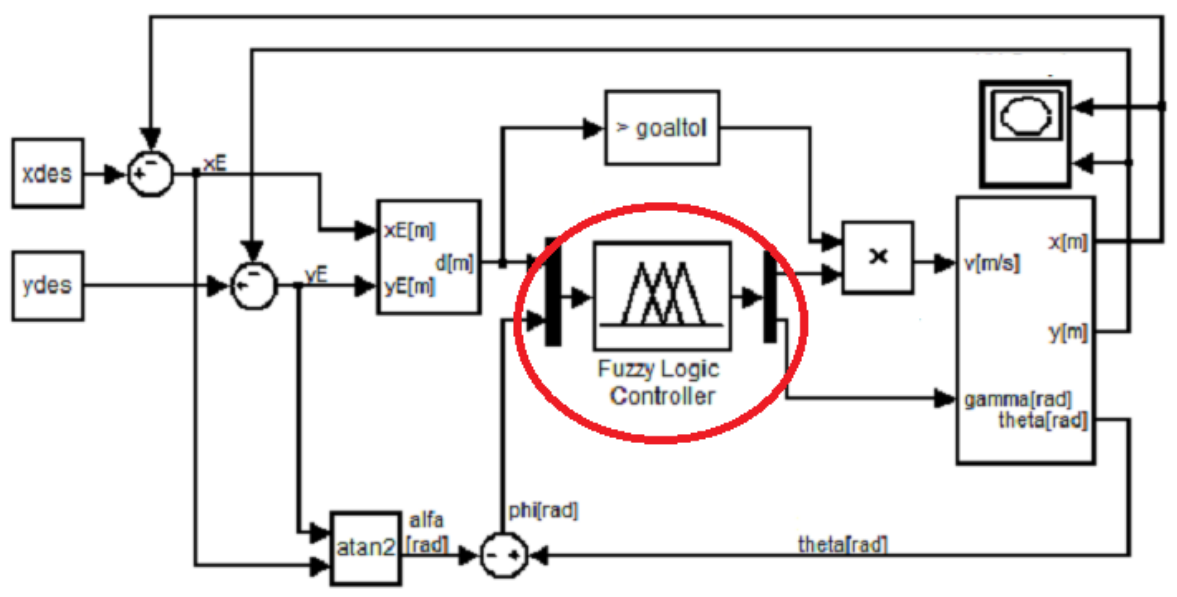

Figura 3 - Controlador Fuzzy desenvolvido neste trabalho.

Embarcar o controlador em um microcontrolador: A evolução da eletrônica e da engenharia de software fornece os meios para o desenvolvimento dos sistemas de automação distribuídos. Componentes com alto desempenho tais como microcontroladores, sensores e atuadores têm sido fabricados a um custo suficientemente baixo para possibilitar a criação de dispositivos autônomos inteligentes [20]. Portanto, embora seja uma tarefa difícil é executável embarcar um controlador fuzzy em um microcontrolador. Para isso os autores desenvolveram o algoritmo de controle fuzzy na linguagem de programação do microcontrolador.

A Figura 4 mostra o sistema de direção assistida proposto neste trabalho já embarcado e integrado com barramento CAN. Consiste de um módulo de controle microcontrolado com comunicação via barramento CAN que executa um algoritmo de controle baseado em lógica fuzzy para acionar o motor elétrico (atuador) instalado na barra de direção. As entradas deste controlador fuzzy são a velocidade do veículo e a rotação do motor que chegam através da rede CAN. Também são entradas do controlador fuzzy o sensor de ângulo de direção e sensor de torque, ambos instalados na estrutura mecânica do sistema de direção.

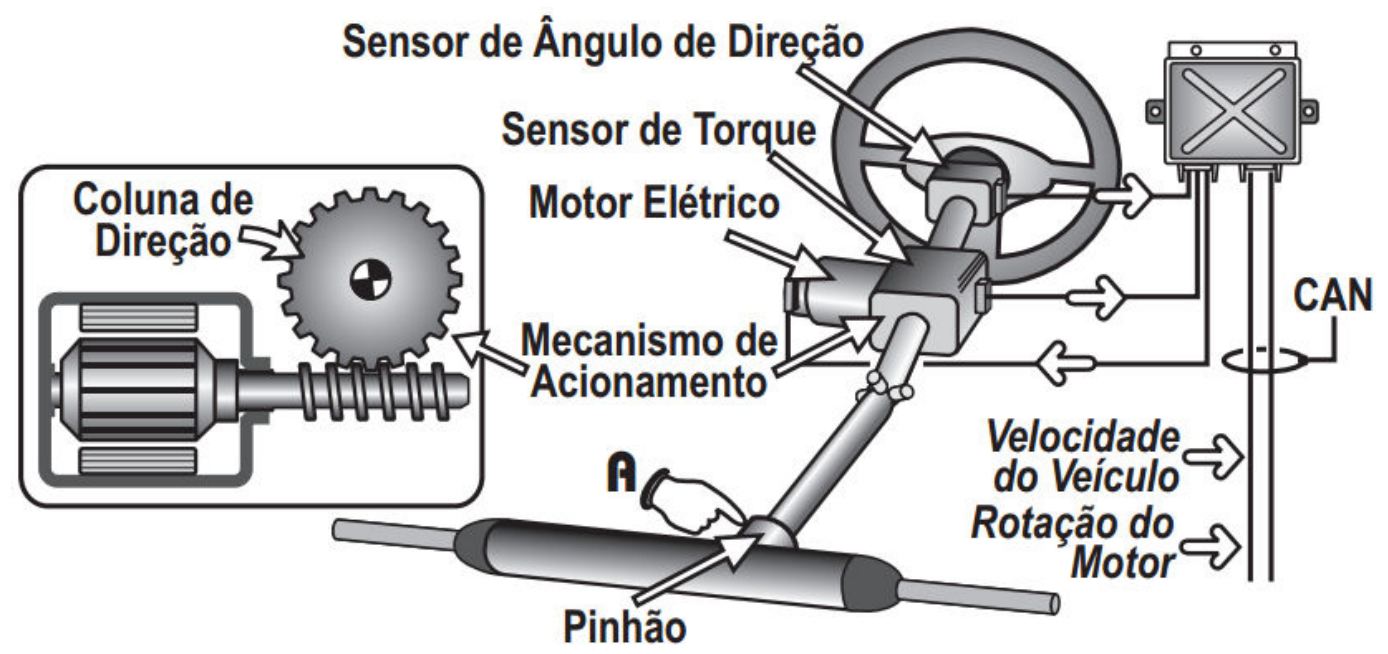

Figura 4 - Controlador Fuzzy e Componentes do EPS. Fonte: [7]. 
Neste trabalho o veículo será modelado por um modelo clássico como mostrado na Figura 5. Neste o veiculo com quatro rodas sendo as duas traseiras fixadas e as duas dianteiras com um grau de liberdade para rotação em torno do eixo $Z$ vertical dando origem ao esterçamento das rodas e mudança da direção do veículo. Também neste modelo é considerado a distancia entre eixos e o centro de rotação instantânea. A postura do veiculo é definida pelo vetor $q=(x, y, \theta)$. A velocidade do veículo é definida como nula na direção y desde que não deslizamento lateral. Portanto tem-se:

$$
V_{i}=v \quad V_{y}=0
$$

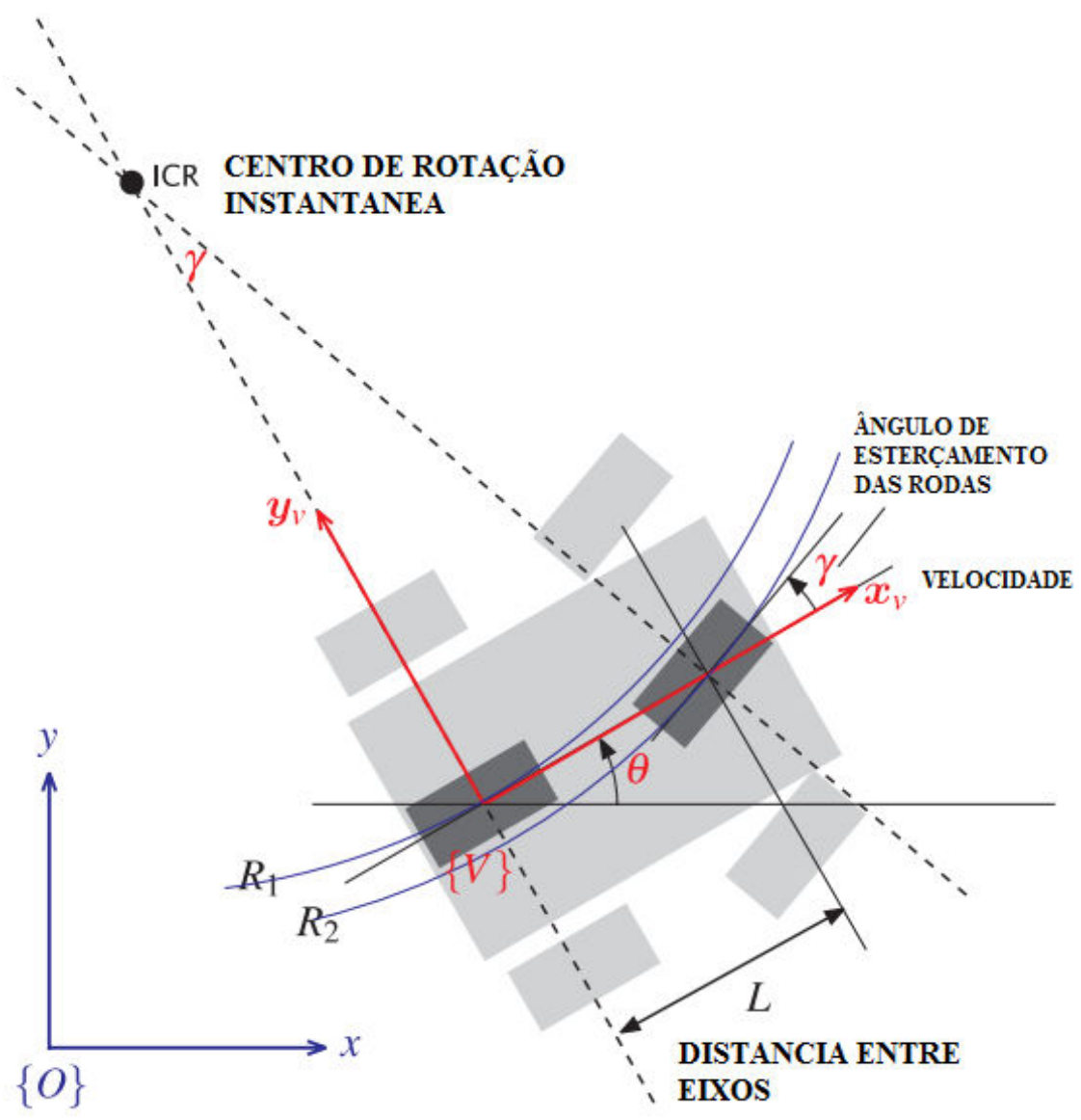

Figura 5- Modelo da cinemática do veículo.

Partindo destas definições a velocidade angular em uma trajetória circular é obtida da seguinte maneira:

$\dot{\theta}=\frac{v}{R_{1}}$

Usando conceitos de geometria é definido $\mathrm{R} 1=\mathrm{L} / \tan \gamma$. Onde o ângulo $\gamma$ é o ângulo de esterçamento das rodas e seus limites definem o valor de R1. A velocidade do veiculo em relação a um referencial inercial considerando a expressão (1) é descrita da seguinte maneira: 


$$
x=v \cdot \cos \theta
$$

$y=v \cdot \operatorname{sen} \theta$

$\dot{\theta}=\frac{v}{L} \cdot \tan \gamma$

Este conjunto de equações descreve a cinemática do veículo. Este modelo será implementado em bloco para simular os movimentos considerando sua velocidade linear, velocidade angular e trajetórias executadas no tempo. A Figura 6 é mostrado a implementação do modelo.

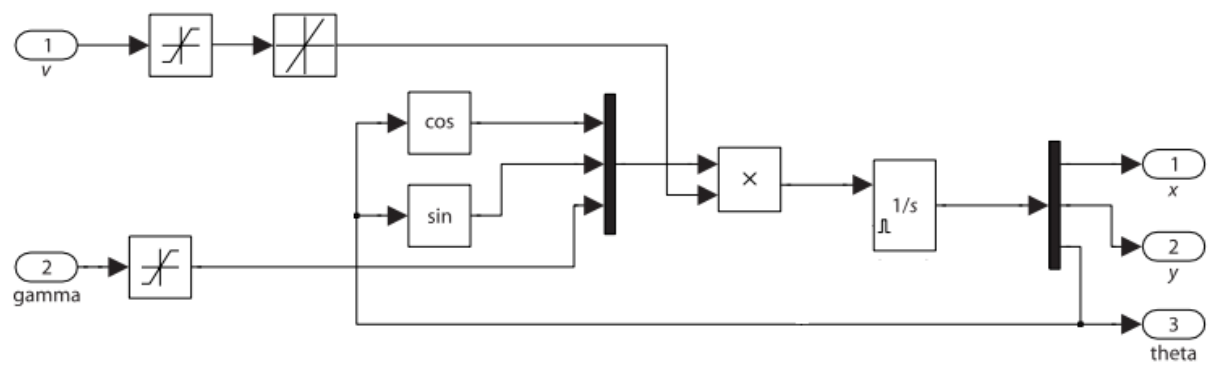

Figura 6 - Modelo da Cinemática Veicular.

Este modelo será incrementado com a inserção do modelo do sistema de direção elétrica. $\mathrm{O}$ modelo adotado para a direção a direção elétrica é o adotado foi desenvolvido em [14] [21] e [22] mostrado na Figura 7.

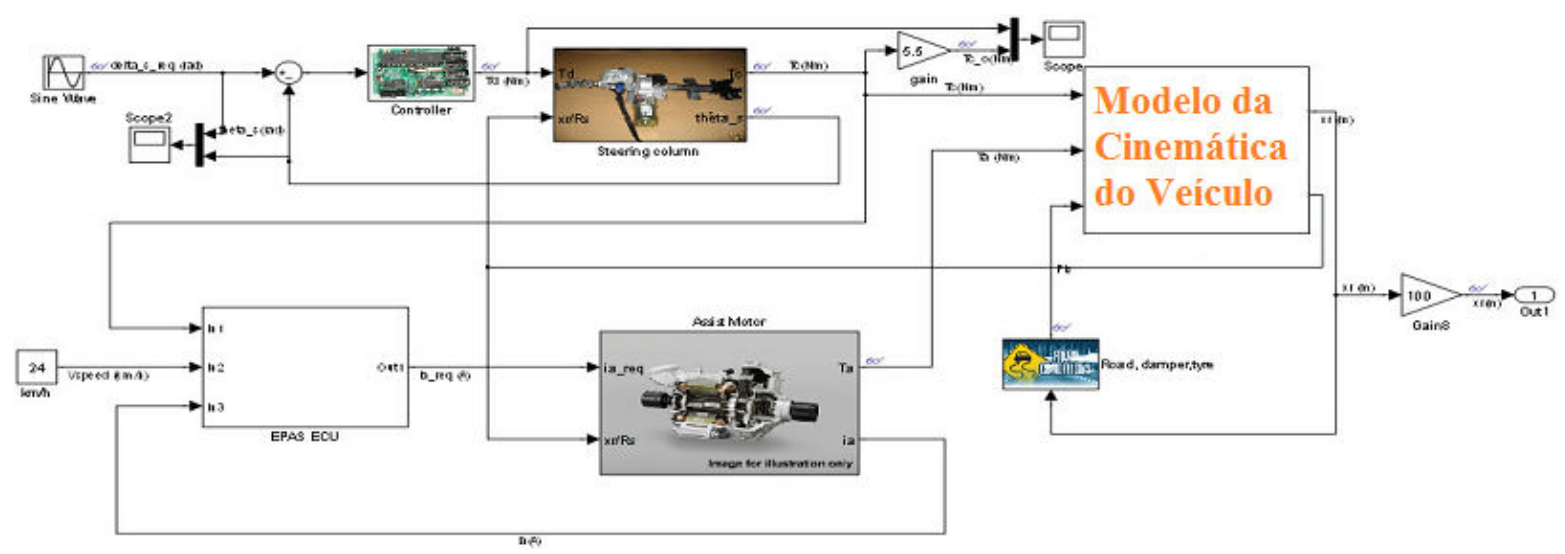

Figura 7- Modelo da dinâmica do sistema de direção elétrica

Este modelo é denominado modelo dinâmico do veiculo por considerar os torques que deram origem aos movimentos realizados no sistema de direção durante a execução da trajetória desejada. As simulações foram realizadas considerando velocidades constantes com objetivo de verificar a curva de operação do torque de direção para cada velocidade desenvolvida pelo veículo. 


\section{SIMULAÇÕES E RESULTADOS}

Simulação computacional: com o modelo do sistema de direção (planta) e um controlador fuzzy será executada o controle em malha fechada [14]. Nesta etapa serão definidos parâmetros e medidas de erro para analisar o desempenho da solução proposta. Uma importante referência nesta etapa da do desenvolvimento da tese é [11] que apresenta um conjunto de dados de cenário urbano brasileiro selecionado e um benchmark de detecção de rodovias composto por dados coletados de sensores tipo RADAR, LIDAR (Light Detection And Ranging) e câmera. Portanto, este trabalho fornece cenários desafiadores para a avaliação do controle de direção fuzzy desenvolvido.

A Figura 8 mostra o modelo do veiculo com o controlador baseado em lógica fuzzy. Com esta estratégia de controle em malha fechada é possível simular o controlador fuzzy para o sistema de direção elétrica.

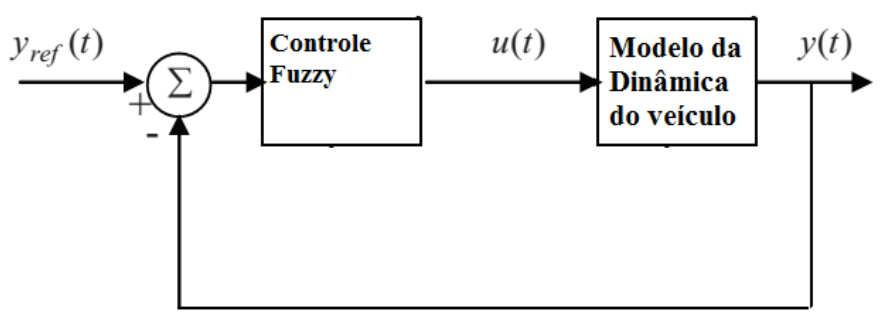

Figura 8 - Controlador Fuzzy em malha fechada.

Com o objetivo de comparar o desempenho do controlador fuzzy com um controlador clássico na Figura 9 é mostrada o modelo da dinâmica do veículo com direção elétrica com um controlador clássico PID em malha fechada. Este controlador teve seus parâmetros ajustando pelo método de ziegler-Nichols [13].

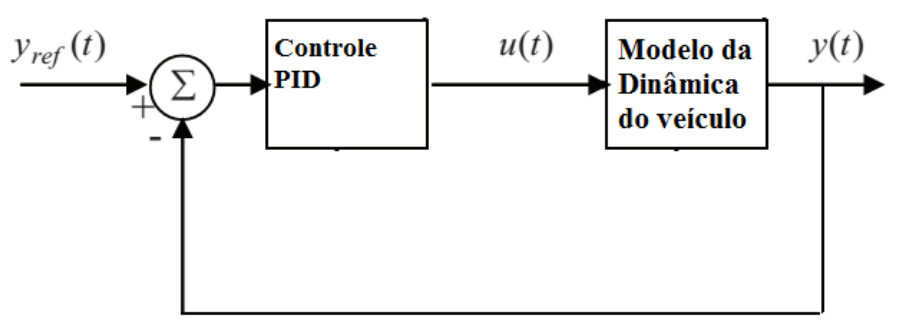

Figura 9 - Controlador PID em malha fechada.

Como o observado nos gráficos das simulações a respostada transitória do controlador fuzzy foi melhor que o PID apresentado um menor sobressinal e menor tempo de resposta. A resposta no regime estacionário do controlador fuzzy também foi melhor, pois minimizou o erro permanente para valores aceitáveis como zero. O gráfico da Figura 10 mostra a resposta ao degrau unitário que representa uma mudança brusca no ângulo de esterçamento das rodas. 


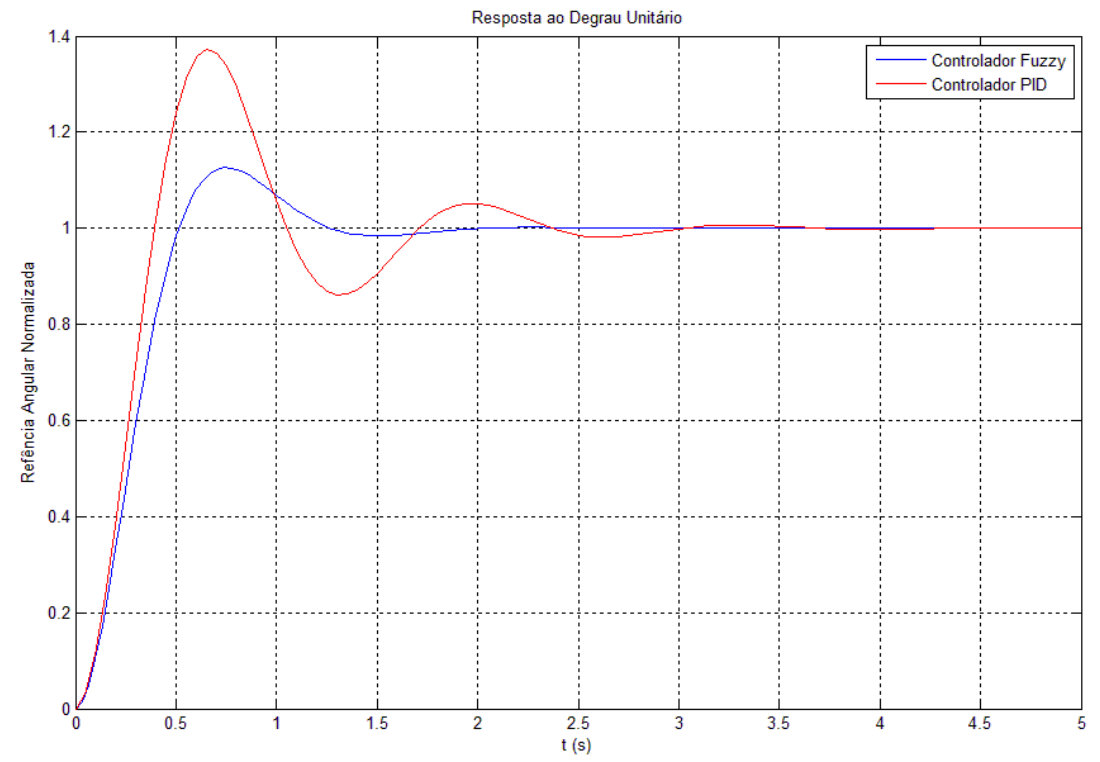

Figura 10 - Entrada tipo degrau unitário para o sistema de direção.

A Figura 11 é mostrada a resposta a uma entrada tipo rampa unitária, que representa uma variação suave, mas contínua no ângulo de esterçamento das rodas. Nesta última simulação a resposta do controlador PID apresentou um erro em regime permanente menor que o controlador fuzzy, porém a grande oscilação gerada pelo PID inviabiliza sua aplicação. $\mathrm{O}$ controlador fuzzy apresentou um erro maior e constante, mas sem oscilações. Esta característica indica a necessita de um ajuste nos parâmetros do controlador fuzzy antes de ser embarcado em um véiculo.

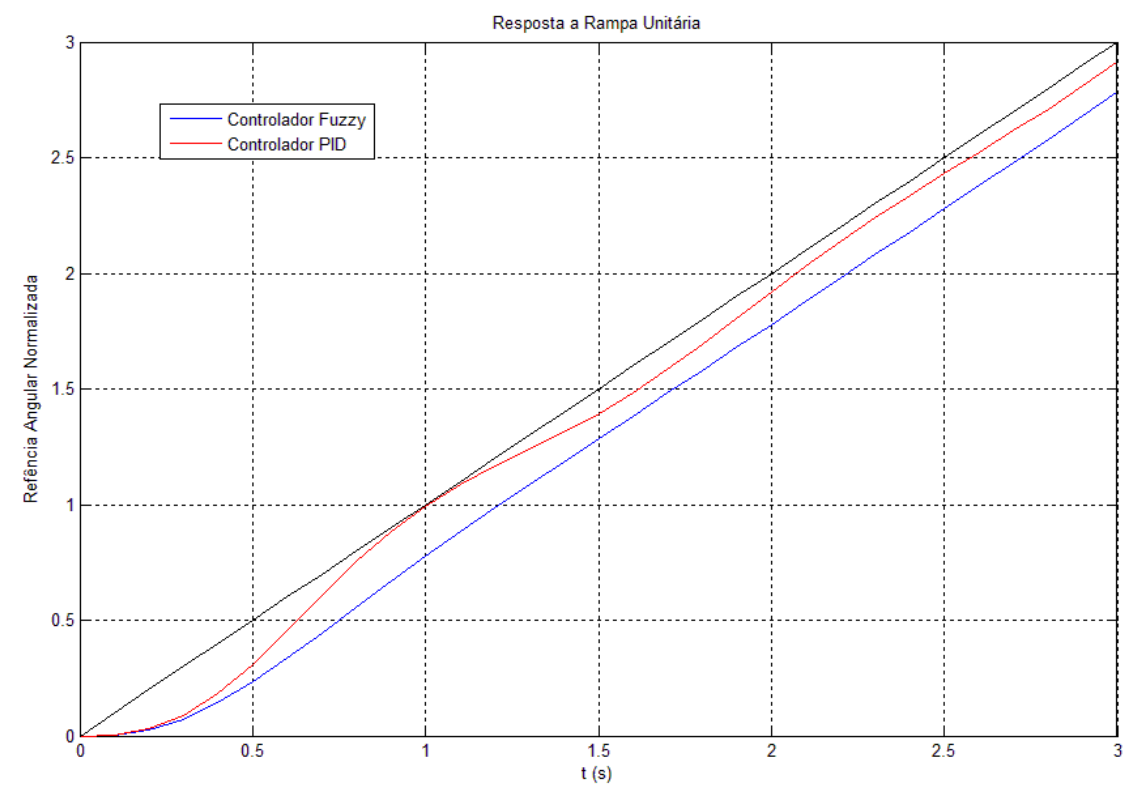

Figura 11 - Entrada tipo rampa unitário para o sistema de direção. 


\section{CONCLUSÕES}

Neste trabalho foi proposto um controlador baseado em lógica fuzzy para um sistema de direção elétrica assistida (EPAS). O desempenho do controlador se mostrou satisfatório nas simulações computacionais quando comparado com o controlador clássico PID. Outra característica importante descrita no trabalho é a integração através de uma rede CAN deste controlador com os demais módulos de controle embarcados no veículo. Portanto, baseados nesses primeiros resultados, os autores entendem que o controlador proposto poderá ser aplicado em carros convencional com sistema de direção elétrica e em carros com qualquer nível de navegação autônoma.

Entretanto mesmo com resultados promissores, a pesquisa está em uma fase inicial visando desenvolver uma solução viável para o problema de navegação autônoma. A próxima fase inclui concluir a conversão da bancada de direção eletro-hidráulica em uma bancada de direção elétrica, ajustar os parâmetros do controlador fuzzy para obter um desempenho ótimo e na fase final embarcar em um veículo real o controlador de sistema de direção elétrica baseado em lógica fuzzy.

\section{REFERÊNCIAS}

[1] MATARIC, M., Introdução à Robótica, 1 ed., São Paulo: Editora Unesp/Blucher, 2014.

[2] ROMERO, Roseli A. F., Robótica Móvel, 1 ed., Rio de Janeiro: LTC, 2014.

[3] NASCIMENTO JÚNIOR, C.L.; YONEYAMA, T., Inteligência Artificial em Controle e Automação, São Paulo: Blucher: FAPESP, 2004.

[4] Hu, P., J Guo, L.Li, and R. Wang, A Robust longitudinal sliding-mode controller design for autonomous ground vehicle based on fuzzy logic, Internatio JournInternational Journal of Vehicle Systems Modelling and Testing, Vol 11, No 4, pp. 368-383, 2013.

[5] Guo, J., L. Li and K. Li, An adaptive fuzzy sliding lateral controle strategy of automated vehicles based on vision navigation, Vehicle System Dynamics, Vol. 51, no. 10, pp. 15021517, 2013.

[6] Fernandes, Leandro C., Wolf, Denis F., CARINA Inteligente Robotic Car: Architectural design and applications, Journal of Systems Architecture, vol. 60, pp. 372-392, 2014.

[7] Manavella, H. J., Eletrônica Embarcada Veicular - Transmissão Automática, ABS/TC/ASR, Suspensão Pilotada, Direção Dinâmica, Estabilidade Eletrônica Veicular. Obra registrada na Fundação Biblioteca Nacional com o número de registro 678.299, 2016.

[8] Campbell, Mark, Magnus Egerstedt, Jonathan P. How, And Richard M. Murray. Autonomous driving in urban environments: approaches, lessons and challenges. Philosophical Transactions of the Royal Society A: Mathematical, Physical and Engineering Sciences 368, no. 1928, 2010.

[9] Deshpande, Pawan. Road Safety and Accident Prevention in India: A review, Internation Journal Adv Engg Tech/Vol. V/Issue II/April- June 64 (2014): 68.

[10] Capelli, Alexandre, Eletroeletrônica Automotiva: injeção eletrônica, arquitetura do motor e sistemas embarcados. São Paulo, Erica, 2010.

[11] Shinzato, Patrick Y.; Wolf, Denis F., CaRINA dataset: An emerging-country urban scenario benchmark for road detection systems, IEEE 19th International Conference on Intelligent Transportation Systems (ITSC), 2016.

[12] Santos, Max M. D., Redes de comunicação automotiva: características, tecnologias e aplicações. São Paulo, Erica, 2010. 
[13] Aguirre , L. A. (ED.). Enciclopédia de Automática: Controle e Automação. 1. ed. São Paulo: Blutcher, Sistemas Lineares e Sistemas Não-lineares, Vol. I,Vol. II e Vol. III 2007.

[14] HASSAN, Mohad K.; AZUBIR, Nor A. M.; Optimal Design of Electric Power Steering System (EPAS) Using GA-PID Method. International Symposium on Robotics Intelligent Sensors, page 614-621, 2012.

[15] Hari, V. M., Lakshmi, P., Design and implementation of Adaptive Neuro Fuzzy Inference System for an experimental Active Suspension System, International Conference on Robotics, Automation, Control and Embedded Systems (RACE), 2015.

[16] Lin, Hui; Song, Chuanxue, Design of a fuzzy logic controller for ABS of Electric Vehicle based on AMESim and Simulink, International Conference on Transportation, Mechanical, and Electrical Engineering (TMEE), 2011.

[17] Massera Filho, C.; Wolf, D. F., Driver Assistance controller for tire saturation avoidance up to the limits of handling, $12^{\text {th }}$ Latin American Robotics Symposium and $3^{\text {rd }}$ Braziliam Symposium on Robotics (LARS-SBR), IEEE, pp. 175-180, 2015.

[18] Silva, José L. N., Projeto e Realização de Controle Fuzzy para manipulador industrial tipo SCARA e sua avaliação de desempenho à luz da norma ISO 9283, Dissertação de mestrado, Universidade Federal do Ceará, Programa de Pós-graduação em Engenharia de Teleinformática, Fortaleza, 2016.

[19] Erdal, K., K. Erkan, R. Herman, and K. Okyay, Towards agrobots: Trajetory control of an autonomous tractor using type-2 fuzzy logic controllers, IEE/ASME Transactions on Mechatronics, vol. 20, no 1, pp. 2912-2924, 2015.

[20] Behera, Laxmidhar, Kar, Indrani, Intelligent Systems and Control: Principles and applications, Oxford University Press, Fifth impression 2014.

[21] CHEN, Hao; YANG, Yali. Study on Electric Power Steering Based on ADAMS and MATLAB. Journal of Software Engineering: page 868-876, 2015.

[22] GUODONG, Yin; ZHENYU, Wang. Active Steering of Autonomous Vehicle Using Model Predictive Control with Legendre Function. $28^{\text {th }}$ Chinese Control and Decision Conference, 2016. 